\title{
Plant callus response to egg-laying by chorus cicada and its effect on egg hatch
}

\author{
C.A. Rowe ${ }^{1}$, D.P. Logan² and P.G. Connolly ${ }^{2}$ \\ ${ }^{1}$ The New Zealand Institute for Plant \& Food Research Ltd, \\ 412 No. 1 Road, RD2, Te Puke 3182, New Zealand \\ ${ }^{2}$ The New Zealand Institute for Plant \& Food Research Ltd, \\ Private Bag 92169, Auckland 1142, New Zealand \\ Corresponding author: Christina.Rowe@plantandfood.co.nz
}

\begin{abstract}
Chorus cicada, Amphipsalta zelandica (Biosduval), is an endemic species of cicada that is a pest of kiwifruit. Adult female cicadas cause damage to kiwifruit canes when egg laying. The oviposition wound often stimulates the local formation of callus tissue in kiwifruit canes. The effect of egg-nest callusing on the proportion of eggs to hatch successfully was investigated as a potential plant resistance trait for selection. The proportion of callus tissue covering the eggnest and the number of eggs hatched were measured from egg-nests in canes of two kiwifruit cultivars, 'Hayward' and 'Zesy002', and stems of 'Hass' avocado. Egg-nests with more callus tissue had reduced egg hatch in both cultivars of kiwifruit and avocado. The host plant also affected egg hatch, with significantly different hatch rates between the two kiwifruit cultivars and avocado at zero percent callusing.
\end{abstract}

Keywords Amphipsalta zelandica, egg-nest callusing, egg hatch, kiwifruit, avocado

\section{INTRODUCTION}

Adults of the chorus cicada species, Amphipsalta zelandica (Boisduval, 1835) (Hemiptera: Cicadidae), are a pest of kiwifruit which emerge en masse in the latter half of January and cause damage and disruption in kiwifruit orchards. Adults of a second cicada species, the clapping cicada $A$. cingulata are a minor pest as they are less abundant (Logan \& Connolly 2005; Logan et al. 2011). Adult female chorus cicadas lay their eggs into current-season canes, creating a wound that develops into a herring-bone shape recognised as a cicada egg-nest. Female cicadas have an average fecundity of 278.5 eggs and have the potential to lay several egg-nests each, based on the average egg-nest size of 79.4 eggs (Logan et al. 2014). The oviposition wound changes the structural integrity of the cane and cane breakage can occur at the site of the egg-nest when the canes are tied down in winter in preparation for the next growing season (Logan \& Connolly 2005). The herringbone shape of a cicada egg-nest is created by the ovipositor which has serrations near its apex and bores a series of paired tunnels at opposite angles into the cane. A batch of eggs is deposited 
at the bottom of each tunnel, which we will refer to as an egg stack. Nymphs begin hatching from the end of May until late December in New Zealand (Logan et al. 2014). After hatching from the chorion, the nymphs make their way through the tunnel, emerge from the nest and then drop to the ground to seek access to a root for feeding.

Cicada oviposition was observed to stimulate the growth of callus tissue at the site of the egg-nest wound in kiwifruit and other host plants on the research orchard. Elsewhere, cicada oviposition has also been reported to cause plant callusing and resin production at the site of the egg-nest wound in various host plants (White 1981; White et. al. 1982; Karban 1983). Callus tissue development on cicada egg-nests can physically encase eggs resulting in mortality (White 1981). The aim of this investigation was to determine how callus tissue development affects egg hatch and nymph emergence in canes from two commonly-grown varieties of kiwifruit (Actinidia chinensis var. deliciosa 'Hayward' and Actinidia chinensis var. chinensis 'Zesy002' (commonly known as Gold3) and stems from another locally grown crop, Persea americana 'Hass' avocado. Callus tissue growth in response to cicada oviposition was expected to have a genetic basis and to differ between plant species and varieties. Quantifying the effect of callus growth to cicada egg-nests may lead to the development of selection criteria for resistance to cicadas in kiwifruit.

\section{MATERIALS AND METHODS}

A preliminary experiment was carried out to determine how callus growth affects cicada hatch in 'Hayward' kiwifruit. Cicada egg-nests $(n=31)$ with a range of callus tissue development were selected from 'Hayward' vines in a block at the Plant \& Food Research orchard in Te Puke. These cicada egg-nests were pruned off in August 2013.The amount of callus tissue covering each egg-nest was measured and the number of eggs which hatched successfully, as determined by empty egg-cases, or dead eggs/nymphs was recorded. Egg-nests were photographed before and after callus tissue was removed from one side (see Figure 1). Based on these photographs, the proportion of callus tissue which covered the egg-nest was calculated using ImageJ software. For each egg stack, the length of the egg stack which was visible in the intact egg-nest was measured (Figure 1a). The total length of the egg stack was measured by removing the callus tissue (Figure $1 b$ ). This was used to calculate the percentage of the egg-nest covered in callus tissue.

To determine the hatch rate for each egg-nest, half the egg-nest was dissected under the microscope (10X) to determine the fate of the eggs. Hatched eggs had an empty white/clear egg case, whereas eggs that failed to hatch were either black or discoloured, sometimes with a shrivelled appearance. Some eggs had hatched successfully but the nymphs had died within the egg-nest. Dead nymphs were included with those eggs that failed to hatch when hatch rate was calculated.

To compare the responses of different plant hosts to cicada oviposition, egg-nests laid in 'Hayward' and Gold3 kiwifruit canes $(n=31$ and 25 respectively), and 'Hass' avocado stems $(n=34)$ were tagged in February 2014 after chorus cicadas had completed egg-laying. Egg-nests laid in Gold3 canes and avocado stems were

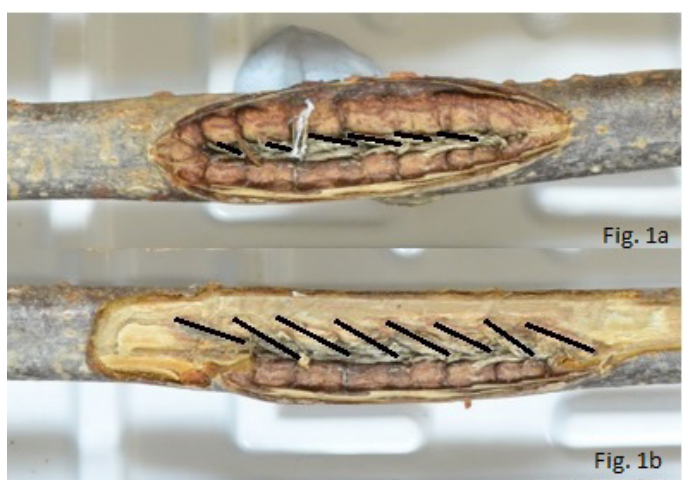

Figure 1a Chorus cicada (Amphipstalta zelandica) egg-nest in Actinidia chinensis var. deliciosa 'Hayward'. Black lines show the measured lengths of egg stacks not covered with callus tissue. Figure 1b Same egg-nest with callus tissue removed. Black lines illustrate the total length of each egg stack. 
Table 1 Quasi-binomial Generalized Linear Model (GLM) coefficients (intercept a and slope b on the logit scale) and their standard errors (SE) for models fitted to percentage callus cover and percentage chorus cicada egg hatch for an preliminary experiment with 'Hayward' kiwifruit and a comparison of three egg-nest hosts, 'Hayward' kiwifruit, Gold3 kiwifruit and 'Hass' avocado.

\begin{tabular}{llcc}
\hline Experiment & Crop & $\mathrm{a} \pm \mathrm{SE}$ & $\mathrm{b} \pm \mathrm{SE}$ \\
\hline Preliminary experiment & 'Hayward' & $2.477 \pm 0.166$ & $-0.051 \pm 0.003$ \\
Host comparison & 'Hayward' & $2.291 \pm 0.311$ & $-0.038 \pm 0.008$ \\
Host comparison & Gold3 & $1.676 \pm 0.392$ & $-0.038 \pm 0.008$ \\
Host comparison & Avocado & $0.285 \pm 0.355$ & $-0.038 \pm 0.008$ \\
\hline
\end{tabular}

sourced from two separate commercial orchards in the Bay of Plenty region. Egg-nests laid in 'Hayward' canes were sourced from the Plant \& Food Research orchard. Egg-nests were left in the canopy for nine months and collected in late November or December 2014 after eggs had hatched (Logan et al. 2014). The cicada egg-nests were stored in the freezer until assessment took place following the method described above.

The relationships between percentage of eggs hatched and the amount of callus tissue for 'Hayward' egg-nests in the preliminary experiment, and for two kiwifruit cultivars and avocado in a second experiment, were modelled by fitting a quasi-binomial Generalized Linear Model (GLM) in R. A quasi-binomial model was used to make allowance for the high degree of

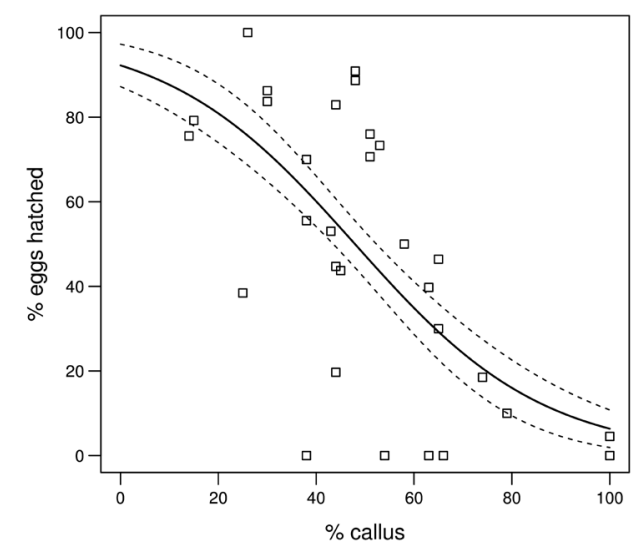

Figure 2 Egg hatch from egg nests laid by chorus cicada, Amphipsalta zelandica, selected for a range of callusing in Actinidia chinensis var. deliciosa 'Hayward' kiwifruit vines. over-dispersion present in the data (dispersion parameters were taken to be 21.6 and 20.1 for the preliminary and host comparison models respectively). From the GLM, coefficients for the equations of the lines for each host were calculated.

\section{RESULTS}

In the preliminary experiment, cicada nymph hatch from 'Hayward' canes was negatively influenced by the percentage of callus tissue $(\mathrm{P}<0.001)$ (Figure 2). Cicada egg-nests with little callusing had a high rate of successful nymph hatch. The minimum percentage of callus cover on an egg-nest was $14 \%$, which produced a nymph hatch of $75 \%$. Egg-nests with $100 \%$ callus cover had 0- 5\% hatch.

In the second experiment, the hatch rate for egg-nests laid in 'Hayward' kiwifruit, Gold3 kiwifruit and 'Hass' avocado also displayed negative relationships with the amount of callusing. The differences between the slopes for the three hosts were not significant $(\mathrm{P}=0.697$ comparing 'Hass' with Gold3; P = 0.287 comparing 'Hass' with 'Hayward'). In this case, a model with three intercepts and a common slope was considered appropriate. The intercepts were significantly different for each host plant $(\mathrm{P}<0.001)$ and new regression equations were fitted to data accordingly (Table 1, Figure 3).

The model co-efficients are given on the logit scale because the GLM with quasibinomial error is a linear regression on the logit transform of $y$, the probability (Table 3 ). Fitted lines in the graphic display, back-transformed from the logit scale, became non-linear and may appear to have different slopes but they do not (Figure 3).

In 'Hass' avocado, mortality was mostly due 


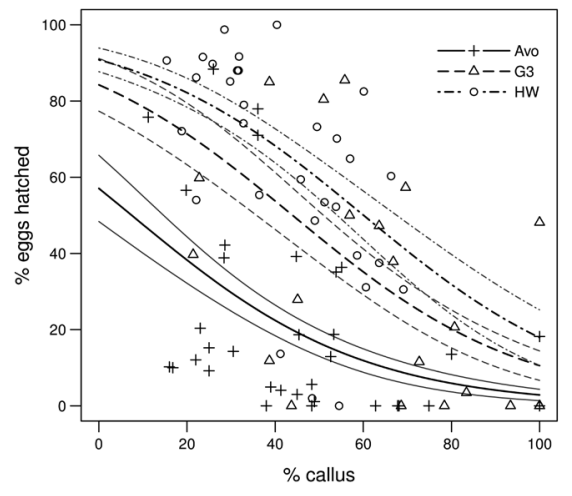

Figure 3 Egg hatch for chorus cicada, Amphipsalta zelandica, compared with percentage of callus tissue covering egg-nests for egg-nests laid in canes of Actinidia chinensis var. deliciosa 'Hayward' (HW), A. chinensis var. chinensis 'Zesy002' (Gold3; G3), and stems of 'Hass' avocado (Avo). Solid lines are backtransformed to percent from logits. The GLM is a linear predictor of the logit transformation of proportion. Dashed lines are back-transformed standard errors.

to death of the egg (97\%, $n=34$ egg-nests). The major cause of mortality in kiwifruit canes was also egg-death, however dead nymphs were also commonly found in 'Hayward' egg-nests (37\% of mortality, $n=20$ egg-nests) and Gold 3 (33\% of mortality, $n=25$ egg-nests) egg-nests.

\section{DISCUSSION}

The aim of this research was to investigate the effect of callus growth on the hatch rate of cicada egg-nests as a basis for the development of selection criteria for resistance to cicadas in kiwifruit. This study found that callus formation was positively associated with reduced cicada egg-hatch for two cultivars of kiwifruit and for 'Hass' avocados. Based on the similarity of the slopes in the GLM, the relationships between callus tissue growth and egg hatch were similar among the host plants (Table 1). Callus tissue development was associated with mortality in cicada egg-nests because of either egg or nymph death. Egg death constituted the largest portion of mortality in all plant cultivars tested. Dead nymphs were found where callus tissue was physically preventing hatched nymphs from emerging from the egg-nest.

The host plant also had a significant effect on egg hatch, as implied by the different intercepts of the regression equations. Most eggs hatched successfully when callus tissue cover was only minor, particularly from egg-nests laid in 'Hayward' and Gold 3, however, fewer nymphs hatched from avocado. At higher rates of callus tissue cover, egg hatch was significantly reduced in all host plants. Egg hatch from egg-nests laid in Gold 3 canes was highly variable and we believe that callus tissue cover may have limited value as a variable for predicting egg hatch for Gold3, and that another variable, such as cane age, may be more influential.

In avocado, there was a strong suppression of egg hatch, even at low rates of callusing. Very few dead nymphs were found in egg-nests dissected from avocado stems, indicating that mortality was occurring earlier during egg development in this host plant. It is possible that mechanism other than callusing may cause cicada egg death. For example, it was found that cherry trees in California have a physiological response to cicada oviposition that reduces egg hatch (Karban 1983). Egg-nests laid in cherry tree stems, which produced a gum exudate at the site of the cicada egg-nest had a reduced hatch compared with egg-nests that did not produce gum. If genetic plant resistance to cicadas is to be developed, then the factors causing cicada egg and nymph death need to be clarified.

A strong callus response may reduce the numbers of cicadas that can complete their lifecycles on a plant host. Cicadas commonly oviposit in both avocado and kiwifruit crops; however, adult cicada emergence within kiwifruit blocks is often much higher than in avocado blocks (D.P. Logan, Plant \& Food Research Ltd, unpublished data). These observations are consistent with the poor egg hatch found in our sample of egg-nests laid in avocado stems. Although the Gold3 samples had a variable hatch 
rate in relation to callusing, our data indicate this cultivar may not support large cicada populations to the same extent as 'Hayward' blocks do currently.

\section{ACKNOWLEDGEMENTS}

Funding for this project was received from the Plant \& Food Research Core kiwifruit research programme. We thank Cathy McKenna and Garry Hill and for improving the text.

\section{REFERENCES}

Karban R 1983. Induced responses of cherry trees to periodical cicada oviposition. Oecologia 59(2-3): 226-231.

Logan D, Connolly P 2005. Cicadas from kiwifruit orchards in New Zealand and identification of their final instar exuviae (Cicadidae: Homoptera). New Zealand Entomologist 28(1): 37-48.
Logan, DP, Hill MG, Connolly, PG, Maher BJ, Dobson SJ. 2011. Influence of landscape structure on endemic cicadas in New Zealand kiwifruit orchards. Agricultural and Forest Entomology 13:259-271.

Logan, DP, Rowe CA, Maher BJ 2014. Life history of chorus cicada, an endemic pest of kiwifruit (Cicadidae: Homoptera). New Zealand Entomologist 37(2): 96-106.

White J. 1981. Flagging: host defences versus oviposition strategies in periodical cicadas (Magicicada spp., Cicadidae, Homoptera). Canadian Entomologist 113:727-738.

White J, Lloyd M, Karban R. 1982. Why don't periodical cicadas normally live in coniferous forests? Environmental Entomology 11: 475482.w 